\title{
Spatially Varying Equilibria of Mechanical Models: Application to Dermal Wound Contraction
}

\author{
LUKE OLSEN, PHILIP K. MAINI ${ }^{\dagger}$ \\ Centre of Mathematical Biology, Mathematical Institute, \\ 24-29 St. Giles, Oxford, OX1 3LB, United Kingdom \\ AND
}

JONATHAN A. SHERRATT

Nonlinear Systems Laboratory, Mathematics Institute, University of Warwick, Coventry, CV4 7AL, United Kingdom

Received 23 December 1996; revised 28 April 1997

\begin{abstract}
Mechanochemical models based on the Oster-Murray continuum framework have been applied to a variety of biological settings to obtain an understanding of the morphogenesis of living tissues. Wound-healing in mammalian skin is an important example, because a complex sequence of biochemical and biomechanical responses are orchestrated to close a wound by a combination of new tissue formation and wound contraction. Mechanical interactions between dermal fibroblastic cells and the collagen-rich extracellular matrix are crucial in the development of a contracted wound state. We and others have previously proposed mechanochemical models for wound repair to gain a greater understanding of both normal and abnormal healing. In the present work, the existence of spatially varying equilibria of these models is investigated by using a small-stain approximation and phase-plane techniques, with numerical simulations to confirm the analytical predictions. These results are sources of novel insight into the roles of key biological parameters in determining the mechanical properties of a contracted wound. These methods may also be relevant to other morphogenetic scenarios for which similar mechanochemical models have been proposed. (C) 1998 Elsevier Science Inc.
\end{abstract}

\section{INTRODUCTION AND BACKGROUND}

The healing of full-thickness excisional skin wounds in adult mammals entails a complex sequence of interregulatory biological processes - see [1] for review - which may be subdivided into the following three temporal phases.

\footnotetext{
${ }^{\dagger}$ Author for correspondence.
} 
1. Inflammation: Platelets and proteins from damaged blood vessels coagulate to form a clot; platelets also release active enzymes and growth factors that attract inflammatory cells into the wound site to degrade necrotic tissue and bacteria.

2. Proliferation: Growth factors also attract dermal fibroblasts into the wound site, which proliferate, release further growth factors, and synthesize new extracellular matrix (ECM)-notably fibrillar collagen, the predominant dermal protein. Fibroblastic cells also generate forces on their substrate by extending cell protrusions, forming cell-surface attachments with collagen fibers and retracting these protrusions [2]. In addition, new blood capillaries develop in the wound from surrounding parent vessels, and the epidermis is healed by epidermal cell migration and proliferation over the provisional wound matrix in the dermis.

3. Remodeling: Fibroblasts in the wound tissue continue to gradually secrete and reorganize the ECM, thereby increasing the mechanical strength of the resultant scar tissue. It appears, however, that this tissue does not fully regain the resilience and architecture of normal dermis and thus remains inferior in quality.

The cell-derived forces generated during wound healing may be beneficial in reducing the wound size by contraction, but are also detrimental because of the high mechanical stresses in and around the scar that can cause pain, disfigurement, and loss of tissue function (especially if located over joints) [3]. Moreover, excessive and permanent contractile forces are characteristic of abnormal healing responses such as keloid scarring and other fibrocontractive diseases [4]. Clinical management of both normal and abnormal wounds may benefit from a greater understanding of the biological mechanisms of dermal wound contraction.

In this paper, we illustrate an analytical approach toward predicting the existence and mechanical characteristics of equilibria of continuum mechanical models of the type originally devised by Oster, Murray, and colleagues for cell traction-driven tissue morphogenesis [5,6]. We have recently proposed a detailed model for the proliferative phase of dermal healing based on this mechanical framework, focusing on how growth factor-mediated cell-ECM interactions lead to wound closure by new tissue formation and contraction [7]. The analytical methods presented in this paper may be applied to our model, thereby enhancing understanding of the mechanical properties of a contracted dermal wound and the roles of key biological parameters.

\section{THE MECHANICAL MODEL FRAMEWORK}

Oster, Murray, and colleagues proposed a continuum model framework in which the basic variables are cell density, $n(\underline{r}, t)$, and ECM 
density, $\rho(\underline{r}, t)$; these are locally averaged species variables that depend on space, $\underline{r}$, and time $t[5,6]$. The mechanical consequences of the cell-ECM traction forces, the intrinsic response of the tissue and the external resistance to tissue movement due to fibrous attachments to underlying tissues, are encapsulated by a force-balance equation that governs the tissue displacement, $\underline{u}(r, t)$. Specifically, in one spatial dimension-say, $x$-the model equations have the form

$$
\begin{aligned}
& \text { cell movement: passive convection }+ \\
& \frac{\partial n}{\partial t}+\overbrace{\frac{\partial}{\partial x}\left[n \frac{\partial u}{\partial t}+\chi(\rho) n \frac{\partial \rho}{\partial x}-D(\rho) \frac{\partial n}{\partial x}\right]}^{\text {haptotaxis (biased) +haptokinesis (unbiased) }}=\overbrace{P(n, \rho)}^{\begin{array}{c}
\text { cell } \\
\text { proliferation }
\end{array}}, \\
& \frac{\partial \rho}{\partial t}+\overbrace{\frac{\partial}{\partial x}\left(\rho \frac{\partial u}{\partial t}\right)}^{\begin{array}{c}
\text { passive } \\
\text { convection }
\end{array}}=\overbrace{B(n, \rho)}^{\begin{array}{c}
\text { ECM biosynthesis }+ \\
\text { degradation by cells }
\end{array}}, \\
& \frac{\partial}{\partial x}[\overbrace{\mu \frac{\partial^{2} u}{\partial x \partial t}}^{\begin{array}{c}
\text { viscous } \\
\text { forces }
\end{array}}+\overbrace{E \frac{\partial u}{\partial x}}^{\begin{array}{c}
\text { elastic } \\
\text { forces }
\end{array}}+\overbrace{\tau(n, \rho)}^{\begin{array}{c}
\text { cell-derived } \\
\text { traction forces }
\end{array}}]=\overbrace{F(\rho, u)}^{\begin{array}{c}
\text { festoring } \\
\text { forces }
\end{array}} .
\end{aligned}
$$

In the specific case of the mechanical responses in wound healing, the model possesses the following particular features:

- Fibroblastic cells are assumed to proliferate according to the logistic growth law: thus, $P=m(1-n)$, where the linear growth rate $r>0$ is assumed to be independent of the collagen density, $\rho$, and the normal dermal fibroblast density is set to unity by the scaling for $n$.

- We neglect the haptotactic contribution to cell movement and assume that random locomotion is independent of $\rho$, under the proviso that $\rho$ is nonzero so that a substrate exists for cell movement; hence, $D>0$ is a constant. These simplifications facilitate the analysis presented herein by decoupling $\rho$ from Equation (1) at equilibrium.

- The rates of collagen biosynthesis and degradation by fibroblastic cells are assumed proportional to $n$ and $-n \rho$, respectively, and the normal dermal collagen density is also scaled to unity. We take the functional form $B=\epsilon n(1-\rho)$, with the parameter $\varepsilon$ satisfying $0<\epsilon \ll$ 1 , to reflect the observation that the rate of ECM remodeling takes place on a relatively long time scale compared with the proliferative phase $[5,7,8]$. 
- A simple linear viscoelastic model is used for the constitutive relation of the tissue that characterizes its response to the cell-derived traction stresses. In reality, a more detailed nonlinear or quasi-linear stress-strain model would be more accurate for cell-collagen composite tissues [9]. This linear form is mathematically valid only for small tissue strains; that is, for $|\partial u(x, t) / \partial x| \ll 1[10]$. The positive parameters $\mu$ and $E$ quantify the viscous and elastic contributions, respectively.

If stress and strain are given by $\sigma$ and $\varepsilon$, respectively, then linear elasticity is modeled by the linear (Hookean) spring, giving $\sigma \sim \varepsilon$, and linear viscosity by the linear (ideal) piston (or dashpot), giving $\sigma \sim \dot{\varepsilon}$, where denotes the time derivative. Note that $\varepsilon=\partial u / \partial x$ in one dimension. The simplest linear viscoelastic models are a combination of a linear spring and a linear dashpot. If the spring and dashpot are in series (the Maxwell model), then $\sigma+\dot{\sigma} \sim \dot{\varepsilon}$, whereas, if they are in parallel (the Voigt model), then $\sigma \sim \varepsilon+\dot{\varepsilon}$. The next simplest model is a combination of the Maxwell and Voigt systems, known as the "standard linear model." Fung [9] offers a detailed discussion on constitutive relations in biomechanics. To be consistent with previous mechanochemical models $[5,11]$, we use the Voigt system.

- The cell traction term, $\tau(n, \rho)$, is the central feature of this framework [6]. Given that traction forces depend on adhesion between cell surface receptors and binding sites on collagen fibers, which is a fast process, it is reasonable to assume $\tau \propto n \rho$ [5]. Furthermore, we propose that the ability of a cell to extend and retract protrusions within a collagen substrate is inhibited at relatively high collagen densities; this is supported by experimental data [12]. We therefore include a sigmoidal inhibition term, so

$$
\tau(n, \rho)=\frac{\tilde{\tau}_{0} n \rho}{R^{2}+\rho^{2}}
$$

where $\tilde{\tau}_{0}$ and $R$ are positive constants. An alternative approach would be to model ECM stiffening by assuming a functional form of $E$ that depends on $\rho$. These different approaches cannot be effectively distinguished on the basis of data from collagen gel studies, and we follow previous authors by taking $E$ to be constant and incorporating this effect into the active traction term.

- The external restoring forces are modeled as homogeneous linear "springs," with elastic modulus proportional to the ECM density: thus, $F=\tilde{s} \rho u$, where $\tilde{s}>0$ measures the strength of the ECM attachments to underlying tissues. 
The uniform equilibrium $(n, \rho, u)=(1,1,0)$ represents normal dermis and is locally stable to small temporal perturbations. Whether this equilibrium is stable to spatiotemporal perturbations is unclear, but straightforward linear analysis can determine parameter values under which it will be stable (see Appendix).

The other uniform equilibria, $(0, \rho, 0)$, represent a continuum of acellular states that are always unstable to the introduction of fibroblasts.

\section{NUMERICAL SIMULATION OF WOUND HEALING}

In this section, we demonstrate that the numerical solutions of Equations (1)-(3), together with the stipulated functional forms (as before) and subject to biological relevant end conditions, may simulate normal wound healing.

By defining the initial wound space as $-1 \leq x \leq 1$ and using symmetry at $x=0$ (the wound center), we may restrict attention to the semi-infinite domain $0 \leq x<\infty$. The initial half-wound is set to unity by the scaling for $x$. The boundary conditions are thus

$$
\begin{aligned}
& \frac{\partial n}{\partial x}(0, t)=\frac{\partial \rho}{\partial x}(0, t)=u(0, t)=0 \\
& \text { and } n(\infty, t)=\rho(\infty, t)=1, \quad u(\infty, t)=0 .
\end{aligned}
$$

The initial conditions are

$$
n(x, 0)=H(x-1), \quad \rho(x, 0)=\rho_{i}+\left(1-\rho_{i}\right) H(x-1), \quad u(x, 0)=0,
$$

where the initial ECM density $\rho_{i}$ inside the wound is due to the early, provisional wound matrix, which is low in collagen and satisfies $0<\rho_{i}<$ 1. $H(\cdot)$ is the Heaviside step function.

Provided the uniform dermal equilibrium is locally stable, the numerical solutions of Equations (1)-(3) subject to end conditions as before are observed to ultimately return to this equilibrium (of which the initial wound state is a large perturbation), as expected [7]. Furthermore, when $\epsilon \ll 1$, corresponding to slow ECM remodeling, a quasisteady state may evolve over a transient time scale as shown in Figure 1 and characterized as follows:

- Cell density, $n$, has returned to its normal dermal value (unity) inside the wound due to proliferation and migration.

- Collagen (ECM) density, $\rho$, deviates only slightly from its initial step function profile, owing to convection and biosynthesis, both of which are relatively slow. 

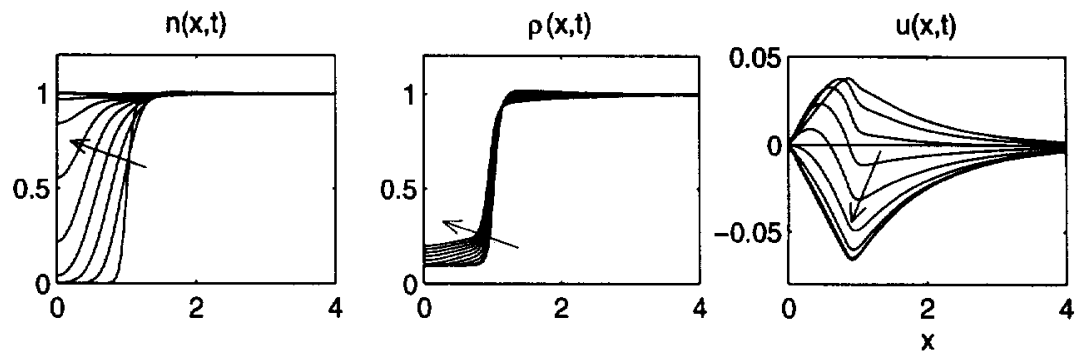

FIG. 1. Numerical solutions of Equations (1)-(3) with functional forms and end conditions as specified in the text, using finite differences for the spatial derivatives and a backward differentiation formula to integrate the resulting system in time (as implemented by the NAG Fortran Library Routine D03PCF). Profiles of fibroblastic cell density $(n)$, collagen density $(\rho)$, and tissue displacement $(u)$ are plotted as functions of distance $(x)$ from the wound center at times $t=0,2,4, \ldots, 20$. A finite domain approximation is used, with zero-gradient boundary conditions at $x_{\infty}=10$. Other parameter values are based on those derived from experimental data and theoretical arguments as given by Olsen et al. [7]: $D=0.01, r=0.5, \epsilon=0.01, \mu=1$, $\tau_{0}=0.1, R=0.2, s=1, \rho_{i}=0.1$, and $p=20$. The initial wound boundary is at $x=1$. The dimensional units of the length and time scales are $1 \mathrm{~cm}$ and 1 day, respectively (for a typical wound). Arrows denote the spatiotemporal evolution of the variables. Note that cells repopulate the wound, collagen gradually fills the wound space, and displacement initially becomes positive and then negative before slowly relaxing back to zero (over a longer time scale - not shown). The initial wound expansion or "gape," is typical of experimental and clinical wounds $[13,14]$.

- Tissue displacement, $u$, becomes nonzero, particularly in and around the wound space. Significantly, $u(1, t)<0$, indicating long-term wound contraction.

This transient time scale is evidently relevant to the proliferative phase of healing when the wound contracts, whereas collagen accumulation in the wound is more intrinsic to the remodeling phase, on a much longer time scale $[7,8]$. To analyze the nature of the contracted quasisteady state, therefore, we seek equilibria of the model for which $\epsilon=0$, so collagen remodeling is neglected. In this case, numerical simulations are similar to the case where $\epsilon$ is small, except that $\rho$ remains permanently close to its initial profile and $u$ evolves to a spatially varying steady profile that may represent a contracted wound state.

To facilitate our mathematical analysis (see Section 4), we use the step function initial profile for $\rho$ as given earlier. In reality, the initial conditions are not of this form, and, for the numerical solution of Equations (1)-(3) as in Figure 1, the initial profiles of $n$ and $\rho$ are approximated by continuous functions so that the cell traction gradient, 
$\partial \tau / \partial x$, in Equation (3) is finite. For example, the sigmoidal forms

$$
n(x, 0)=\frac{x^{p}}{1+x^{p}} \quad \text { and } \quad \rho(x, 0)=\rho_{i}+\left(1-\rho_{i}\right) \frac{x^{p}}{1+x^{p}}
$$

with $p \gg 1$ yield profiles similar to those inferred (later) for the step function initial profiles. To see this, we set $\epsilon=0$ and numerically solve Equations (1)-(3) for different values of $p$. Figure 2 confirms that the steady-state displacement $(u)$ profile is qualitatively invariant with $p$, although the minimum near $x=1$ becomes sharper as $p$ increases. This is expected, because the gradients of the initial profiles for cells $(n)$ and collagen ( $\rho$ ) become steeper about $x=1$ as $p$ increases.

\section{PHASE PLANE ANALYSIS}

We consider Equations (1)-(3) for the case $\epsilon=0$ in Equation (2). We begin the analysis by linearizing Equation (2) about the initial profile. Setting $\rho=\rho_{i}+\rho^{*}$, where $\rho^{*}$ is small, we have the linear approximation $\partial / \partial t\left(\rho^{*}+\rho_{i} \partial u / \partial x\right)=0$. Therefore $\rho^{*}=C-$ $\rho_{i} \partial u / \partial x$, where $C$ is determined by the initial conditions. Hence

$$
\rho \approx \begin{cases}\rho_{i}(1-\partial u / \partial x), & 0 \leq x<1 \\ 1-\partial u / \partial x, & x>1,\end{cases}
$$

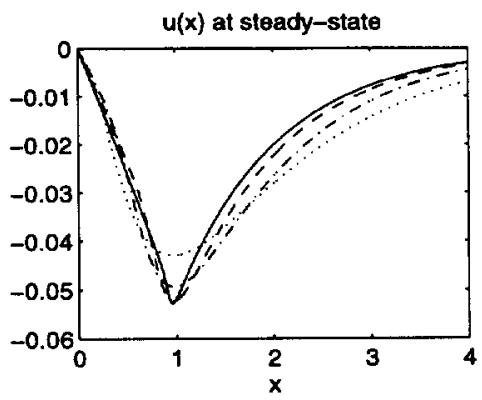

FIG. 2. Numerical solutions of Equations (1)-(3) showing only the steady-state displacement profile, $u\left(x, t_{\infty}\right)$, with end conditions and parameter values as in Figure 1 , except that $\epsilon=0$ and $p=2$ (dotted curve), 4 (dot-dashed curve), 10 (dashed curve), and 50 (solid curve). Recall that $p$ is the Hill coefficient of the sigmoidal curves that approximate the step function initial cell $(n)$ and collagen $(\rho)$ profiles (see text). Here, $t_{\infty}=40$ is used to represent the ultimate steady-state profiles-these are not discernibly different at times greater than $t_{\infty}$. The steady-state displacement profiles become sharper near $x=1$ as $p$ increases. 
as suggested by the small-strain restriction (because the convective flux should be small) and justified by the numerical solutions as discussed earlier.

Equation (1) implies, for our model, that no biological realistic, bounded equilibria exist for $n(x)$ other than the uniform solution $n \equiv 1$; this may be confirmed by seeking such solutions in the $\left(n, n^{\prime}\right)$-phase plane. At equilibria where $n=1$, Equation (3) yields the following boundary value problem:

$$
u^{\prime \prime}+\mathscr{T}(\rho) \rho^{\prime}=s \rho u \quad \text { with } \quad u(0)=u(\infty)=0
$$

where ' denotes $d / d x, \rho$ is proportional to $\left(1-u^{\prime}\right)$ as in Equation (4), $\mathscr{K}(\rho)=\partial \tau(n, \rho) /\left.\partial \rho\right|_{n=1}, \tau_{0}=\tilde{\tau}_{0} / E$, and $\tilde{s} / E$. Solutions of Equation (5) can be studied in the $\left(u, u^{\prime}\right)$-phase plane by casting it in the form

$$
\begin{aligned}
u^{\prime} & =v, \\
v^{\prime} & = \begin{cases}\frac{s \rho_{i} u(1-v)}{1-\rho_{i} \mathscr{T}\left[\rho_{i}(1-v)\right]}, & 0 \leq x<1 \\
\frac{s u(1-v)}{1-\mathscr{T}(1-v)}, & x>1 .\end{cases}
\end{aligned}
$$

The nullclines are $u=0, v=0$, and $v=1$, and the origin, $(u, v)=(0,0)$, is the only fixed point. The eigenvalues of this system imply that the origin is a saddle point or a center, depending on $\mathscr{T}\left(\rho_{i}\right) \lessgtr 1 / \rho_{i}$, respectively, for $0 \leq x<1$ and on $\mathscr{T}(1) \lessgtr 1$ for $x>1$.

Singularities may occur at $\rho=\rho_{s}$ (say) if $\mathscr{T}\left(\rho_{s}\right)=1 / \rho_{i}$ or 1 . At such a singularity, which represents a line $v=v_{s}$ in the $(u, v)$-phase plane, $u^{\prime \prime}$ becomes infinite, whereas $u$ and $\boldsymbol{u}^{\prime}$ are finite. In fact, if a particular solution trajectory encounters the singular line at $x=x_{s}$, then there must be a (finite) jump discontinuity in $u^{\prime}$ with continuity of $u$ at $x_{s}$.

Focusing first on the phase plane for $x>1$, we find that the origin is a saddle point if and only if $\mathscr{T}(1)<1$; that is,

$$
\tau_{0}\left(R^{2}-1\right)>\left(R^{2}+1\right)^{2}
$$

It is a center if and only if $\mathscr{F}(1)>1$, as illustrated in Figure 3 . Note that the condition for the origin to be a saddle point in the $(u, v)$-phase plane is also a necessary (but not sufficient) condition for the uniform equilibrium $(n, \rho, u)=(1,1,0)$ to be stable to small spatiotemporal perturbations (see Appendix). Also, singularities exist at $\rho=\rho_{s}$ given by 
(a)

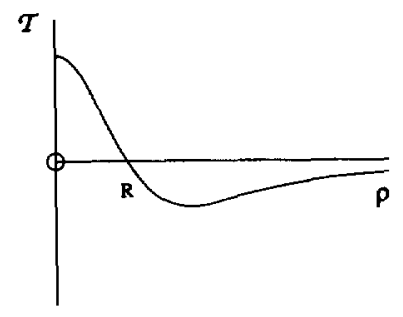

(b)

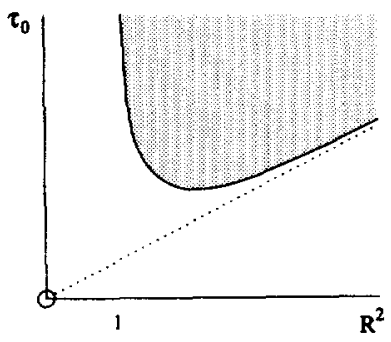

FIG. 3. (a) Plot of the function $\mathscr{T}(\rho)=\tau_{0}\left(R^{2}-\rho^{2}\right) /\left(R^{2}+\rho^{2}\right)^{2}$. (b) The boundary curve $\tau_{0}=\left(R^{2}+1\right)^{2} /\left(R^{2}-1\right)$ between the regions where the origin is a center (shaded) and a saddle (unshaded), for $x>1$, given by the inequality (8). A singularity occurs for some positive value of $\rho$ if $\tau_{0}>R^{2}$, as demarcated by the dotted line.

the positive solutions of $\mathscr{T}\left(\rho_{s}\right)=1$, which is equivalent to

$$
\tau_{0}\left(R^{2}-\rho_{s}^{2}\right)=\left(R^{2}+\rho_{s}^{2}\right)^{2} .
$$

From this, it can be shown that a positive singular value, $\rho_{s}$, exists only if $\tau_{0}>R^{2}$ (see Figure 3) and that is satisfies $0<\rho_{s}<R$.

The various cases for the phase plane structure, according to the values of the parameters $\tau_{0}$ and $R$, are shown in Figure 4 . The small-strain assumption implies $|v| \ll 1$ for a mathematically valid solution; yet, at a singularity, the jump in $v$ cannot be determined a priori-in numerical simulations, this jump depends on the discretization and approximation methods used to integrate Equations (6) and (7) in the vicinity of the singularity. Hence, to ensure a valid solution, $\tau_{0}$ and $R$ must be chosen such that $v_{s}$ is not close to zero; that is, $\rho_{s}$ is not close to unity. This is satisfied by points in $\left(\tau_{0}, R^{2}\right)$-space away from the boundary curve in Figure 3.

$x>1$

The boundary condition $u(\infty)=0$ implies that the origin must be a saddle point as in Figure 4(a,b) for $x>1$, with the solution converging toward the origin along the stable manifold as $x \rightarrow \infty$. If this solution lies in the top-left quadrant of the $\left(u, u^{\prime}\right)$-phase plane, then $u<0$ and $u^{\prime}>0$ represents a contracted tissue displacement profile for $x>1$ (outside the wound). Conversely, if the trajectory lies to the bottom-right quadrant, then tissue expansion is inferred.

By tracing backward in $x$ from infinity along the stable manifold, we find that the solution reaches a point in the $\left(u, u^{\prime}\right)$-phase plane corre- 

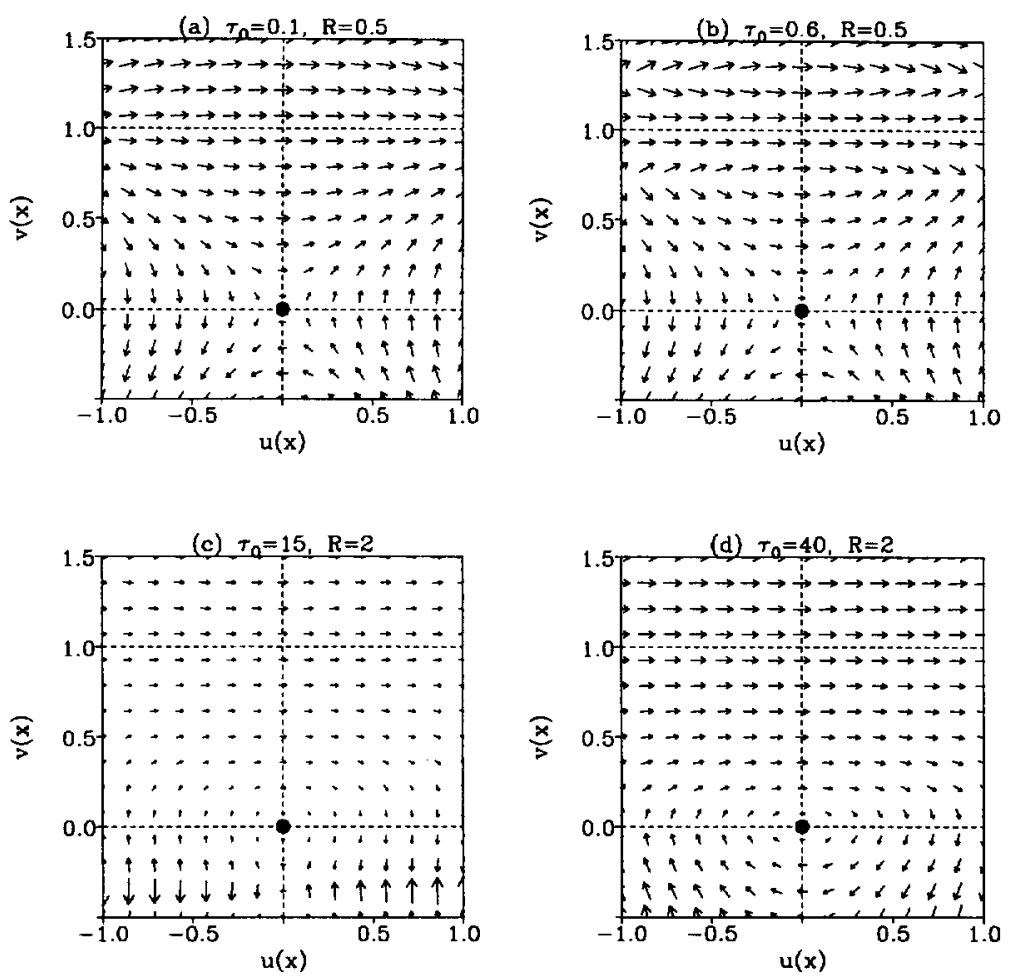

Fig. 4. Phase-plane diagrams for Equations (6) and (7) illustrating the phase flows (arrows) and nullclines (dotted lines) for four different cases of the values of the parameters $\tau_{0}$ and $R$, indicating: (a) $\mathscr{T}(0)<1$, so the origin is a saddle point and no singularities exist; (b) $\mathscr{F}(0)>1$ and $\mathscr{T}(1)<1$, so the origin is a saddle point with a singularity for which $0<\rho_{s}<1$, that is, $0<v_{s}<1$; (c) $\mathscr{g}(1)>1$, so the origin is a center with a singularity for $1<\rho_{s}<R$; (d) similarly, except the singular line $v=v_{s}$ is no longer within the range of the axes shown-this also shows the center at the origin more clearly. Note that singularities actually occur in pairs for our model, at $\rho= \pm \rho_{s}$, but the negative root (corresponding to $v_{s}>1$ ) is not realistic.

sponding to $x=1$ where $u=u_{1}$, say.

$0 \leq x<1$

Continuity of $u(x)$ implies that the solution trajectory for $0 \leq x<1$ must approach the line $u=u_{1}$ as $x \rightarrow 1$ from below. The boundary condition $u(0)=0$ does not specify $u^{\prime}(0)$; rather the "initial point" on the $u^{\prime}$-axis is determined by the preceding continuity condition at $x=1$. For $0 \leq x<1$, it can be shown (using phase-plane arguments analogous 


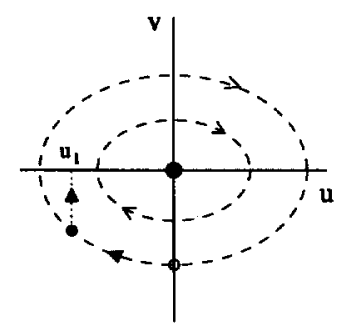

$0 \leq \mathrm{x}<1$

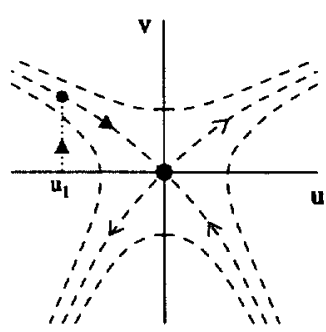

$x>1$

FIG. 5. Qualitative illustration of a possible solution trajectory as described in the text, showing a case in which the origin is a center for $0 \leq x<1$ and a saddle point for $x>1$ with $u(x) \rightarrow 0$ from below as $x \rightarrow \infty$. See also Figure 6(b). Dashed curves denote phase trajectories, with the contracted solution curve highlighted by solid arrows. The dotted line indicates the discontinuity in $v$ (or $u^{\prime}$ ) at $x=1$, where $u=u_{1}$.

to those for $x>1$ ) that the origin may be either a saddle point or a center, depending on the values of $\tau_{0}, R$, and $\rho_{i}$. In either case, a solution trajectory may exist as discussed earlier. If the origin is a saddle point, then the solution in $0 \leq x<1$ is expected to be monotonic either increasing with increasing gradient or decreasing with decreasing gradient. If the origin is a center, then the solution in $0 \leq x<1$ may be oscillatory. Figure 5 illustrates the qualitative construction of such a solution.

Examples of various theoretically possible forms of steady solutions for $u(x)$ are illustrated in Figure 6. We have obtained numerical solutions that yield steady profiles for $u$ resembling those in Figure $6(\mathrm{a}-\mathrm{e})$, depending on the particular parameter values used.

\section{CONCLUSIONS AND DISCUSSION}

The key .conclusion of this work is that, by neglecting collagen kinetics [i.e., setting $\epsilon=0$ in Eq. (2)], we can derive approximations to the collagen profile (in terms of the tissue strain) that yield a boundary value problem governing the tissue displacement that may develop during the proliferative phase of wound healing in excisional skin wounds. Phase-plane techniques applied to this problem (1) inside and (2) outside the wound reveal important details of the nature of these solutions. In particular, a contracted steady state exists only if the cell-derived traction term $\tau(n, \rho)$ is sufficiently insensitive to changes in the ECM density $\rho$ at equilibrium. In addition, the displacement profile inside the wound may be oscillatory or monotonic, depending on 
(a)

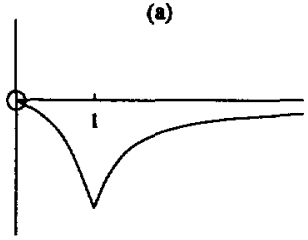

(d)

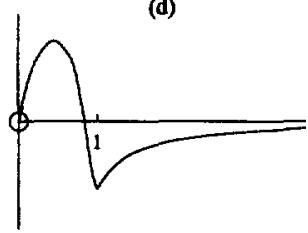

(b)

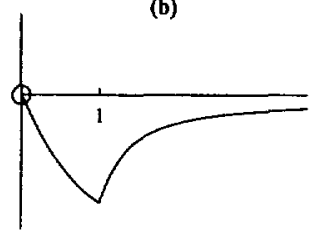

(e)

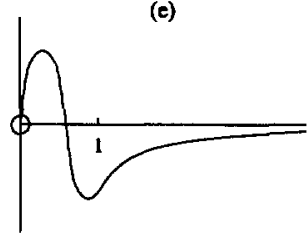

(c)

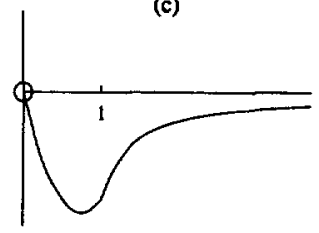

(f)

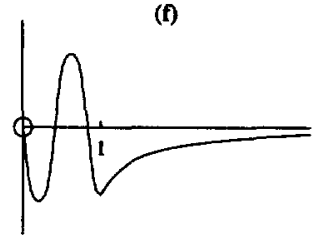

FIG. 6. Possible qualitative forms of the solution $u(x)$ of the boundary value problem (5), representing contracted tissue displacement profiles. The point $\left(u, u^{\prime}\right)=(0,0)$ must be a saddle point for $x>1$ in the $\left(u, u^{\prime}\right)$-phase plane, with $u$ increasing to zero and $u^{\prime}$ decreasing to zero monotonically along the stable manifold in the top-left quadrant as $x \rightarrow \infty$. For $0 \leq x<1$, the origin may be either a saddle point, in which case the profiles for $u$ and $u^{\prime}$ are monotonic decreasing as shown in (a), or a center, in which case $u$ and $u^{\prime}$ oscillate about the origin as shown in (b-f); within this region, any number of oscillations is possible-for example, ( $f$ ) is equivalent to (b) modulo one period. Note that these steady-state profiles but with reversed signs of $u$ and $u^{\prime}$ also are admissible solutions of the boundary value problem (5), representing expanded tissue displacement profiles because $u(1)$ would be positive. Recall that $x=1$ is the initial wound boundary. In numerical simulations of the full system, we have found the profiles (a)-(e) with increasing traction parameter.

the relative magnitudes of the initial ECM density and the traction parameters.

In reality, the rate of fibrillar collagen remodeling, $\epsilon$, is not zero, but the value of this small parameter is difficult to measure in vivo. Taking larger values of $\epsilon$ leads to results quantitatively different from those in Figure 1 (e.g., an increase in collagen density), but the qualitative behavior is preserved. For larger $\epsilon$, the accuracy of our analytic approximation decreases.

The requirement that the uniform equilibrium $(n, \rho, u)=(1,1,0)$ corresponds to a saddle point in the $\left(u, u^{\prime}\right)$-phase plane for $x>1$ implies a restriction on the parameter values in the cell-derived traction stress term, $\tau$, for the existence of a nonuniform solution to the boundary value problem (5). Specifically, if

$$
\tau(n, \rho)=\frac{\tau_{0} n \rho}{R^{2}+\rho^{2}},
$$


then we require

$$
\frac{\partial \tau}{\partial \rho}(1,1)<1 \Rightarrow \tau_{0}\left(R^{2}-1\right)<\left(R^{2}+1\right)^{2}
$$

If this condition is violated, then numerical solutions of the full model system exhibit unbounded instability. Intuitively, this can be understood by the positive feedback effect of the high cell traction gradient around the wound. In this case, the uniform steady state is also unstable to perturbations of arbitrarily small wavelength and therefore model behavior is very sensitive to initial conditions (see Appendix).

Furthermore, singularities in the system may occur at points other than $x=1$, where $u(x)$ is continuous but $u^{\prime}(x)$ is discontinuous, for certain values of $u^{\prime}$. Equations (3) or (5) both illustrate the biological phenomena occurring at these singularities: jump discontinuities in $x$ occur for both the cell traction and the intrinsic elastic stresses-however, these are equal in magnitude and opposite in sign, so the sum of the corresponding forces (the stress gradients) is continuous in $x$ and proportional to the tissue displacement. Because the small-strain assumption used to derive the force-balance Equation (3) imposes the restriction $\left|u^{\prime}\right| \ll 1$, these singularities should occur only if the singular values of $u^{\prime}$ are close to zero. This can be avoided provided that

$$
\tau_{0} \rho_{i}\left(R^{2}-\rho_{i}^{2}\right) \neq\left(R^{2}+\rho_{i}^{2}\right)^{2} \quad \text { and } \quad \tau_{0}\left(R^{2}-1\right) \approx\left(R^{2}+1\right)^{2}
$$

where $\rho_{i}$ is the initial ECM density inside the wound, owing to the provisional matrix deposited during the inflammatory phase of healing.

These analytical methods do not, however, determine whether the nonuniform solution of Equation (5) represents a contracted or expanded tissue, according to $u(1) \lessgtr 0$, respectively. Neither do they predict the nature of the spatially oscillating solution in the region $0 \leq x<1$, which occurs if

$$
\tau_{0} \rho_{i}\left(R^{2}-\rho_{i}^{2}\right)>\left(R^{2}+\rho_{i}^{2}\right)^{2}
$$

They are, however, sources of insight into the dependence of nonuniform tissue displacement profiles on the parameter values in the cell traction term $\tau(n, \rho)$ and into whether these solutions exhibit oscillatory or monotonic features inside the wound space.

Other applications of the foregoing framework may require different functional forms and end conditions. Several authors have proposed mechanochemical models based on cell-ECM interactions for describing morphogenetic events, particularly in embryonic development, as discussed by Murray et al. [11], for example. 
Tranquillo and Murray [15] considered the problem of dermal wound contraction by using a base model similar to that discussed earlier. By imposing a steady chemical profile centered on the wound site, modeling growth-factor mediation of fibroblastic cell growth, traction, and chemotaxis, the model was able to simulate realistic wound contraction. Because of this chemical gradient, however, the analysis presented in this work cannot be extended to their models, and numerical methods are required to solve the nonautonomous boundary value problem for the steady displacement profile.

The more detailed mechanochemical model of Olsen et al. [7] explicitly modeled the dynamics of the growth factors and of a distinct cell population, myofibroblasts. They are phenotypically transformed fibroblasts that are believed to be important in wound contraction and, moreover, in fibrocontractive diseases associated with dysfunctional regulation of the proliferative phase of wound healing $[4,16]$. For the purposes of the work presented in this paper, however, this more complicated model can be analyzed by analogous methods.

Tracqui et al. [17] have recently proposed a novel extension of the Oster-Murray mechanical model to include two distinct ECM types. The first is an early, provisional wound matrix with a linear viscoelastic rheology that is degraded by wound fibroblasts. The second is a subsequent, collagenous matrix with nonlinear elastoplastic properties, which is deposited by fibroblasts and forms the scar tissue. The plasticity of the collagenous ECM effectively "glues" the tissue in place in response to cell-derived traction forces, resulting in permanently contracted displacement and residual stress profiles.

In wound healing, a greater understanding of the physical forces that are associated with wound contraction may lead to improved treatments in the control of scarring $[3,18]$. This possibility has been made more viable by recent tissue culture bioengineering advances enabling quantitative measurements of the mechanical parameters in cell-populated collagen gel assays $[19,20]$, which are in vitro assays that realistically simulate wound contraction $[12,18,21,22]$. The analytical work presented in this paper contributes to this research effort by highlighting explicit relations between the biological parameters that determine the mechanical nature of a healed wound.

\section{APPENDIX}

In this appendix, we consider the linear stability analysis of the uniform equilibrium solution $(n, \rho, u)=(1,1,0)$ to Equations (1)-(3) with $\epsilon$ set to zero (for comparison with the version of the model analyzed in Section 4). Rescaling $\tau$ and $s$ as in Section 4 and seeking 
solutions of the form $(n, \rho, u)=(1,1,0)+\underline{c} \exp (i k x+\lambda t)$, where $\underline{c}$ is a constant vector with $|c| \ll 1$, the dispersion relation is

$$
2 \mu k^{2} \lambda\left(k^{2}\right)=-b \pm\left[b^{2}-4 \mu k^{2} c\right]^{1 / 2}
$$

where

$$
\begin{aligned}
& b\left(k^{2}\right)=\mu D k^{4}+\left(1+r \mu-\tau_{1}-\tau_{2}\right) k^{2}+s \\
& c\left(k^{2}\right)=D\left(1-\tau_{2}\right) k^{4}+\left[D s+r\left(1-\tau_{2}\right)-\tau_{1}\right] k^{2}+r s,
\end{aligned}
$$

and $\tau_{1} \equiv \partial \tau(n, \rho) / \partial n, \tau_{2} \equiv \partial \tau(n, \rho) / \partial \rho$, evaluated at $n=1, \rho=1$.

The uniform steady state will lose stability if $b$ or $c$ become negative: $b$ becomes negative if

$$
\tau_{1}+\tau_{2}>1+r \mu+2(\mu s D)^{1 / 2}
$$

whereas $c$ will become negative if

$$
\tau_{2}>1, \quad \text { or } \quad \tau_{2}<1 \quad \text { and } \tau_{1}-r\left(1-\tau_{2}\right)-D s>2\left[D\left(1-\tau_{2}\right) r s\right]^{1 / s}
$$

Hence, a number of possibilities can arise; see Murray [23]:

- If neither of the foregoing inequalities hold, then the steady state is locally stable.

- If inequality (12) holds but $c$ is nonnegative, then, when $b\left(k^{2}\right)=0$, the steady state undergoes a Hopf bifurcation and the prediction is that there is no steady-state finite amplitude solution.

- If inequality (12) is not satisfied but $\tau_{2}>1$, then $b$ is always nonnegative, but there exists a range $\left[k_{c}^{2}, \infty\right]$ of $k^{2}$ over which $c$ is negative. Hence the steady state will be unstable to perturbations of arbitrarily small wavelengths.

- If both $b$ and $c$ go negative, then the steady state will be unstable, either of the above two behaviors may occur, or the steady state may evolve to a finite amplitude spatially varying steady state.

L. O. expresses gratitude to the Engineering and Physical Sciences Research Council of Great Britain for a Research Studentship award to support this work. We also thank the London Mathematical Society for support. 


\section{REFERENCES}

1 R. A. F. Clark, Biology of dermal wound repair, Dermatol. Clin. 11:647-666 (1993).

2 A. K. Harris, D. Stopak, and P. Wild, Fibroblast traction as a mechanism for collagen morphogenesis, Nature 290:249-251 (1981).

3 R. Rudolph, J. Vande Berge, and H. Paul Ehrlich, Wound contraction and scar contracture. In Wound Healing: Biochemical and Clinical Aspects, I. K. Cohen, R. F. Diegelmann, and W. J. Lindblad, Eds., Saunders, Philadelphia; London, 1992, pp. 96-114.

4 O. Skalli and G. Gabbiani, The biology of the myofibroblast: relationship to wound contraction and fibrocontractive diseases. In The Molecular and Cellular Biology of Wound Repair, R. A. F. Clark and P. M. Henson, Eds., Plenum, New York, 1988, pp. 373-402.

5 G. F. Oster, J. D. Murray, and A. K. Harris, Mechanical aspects of mesenchymal morphogenesis, J. Embryol. Exp. Morphol. 78:83-125 (1983).

6 J. D. Murray and G. F. Oster, Cell traction models for generating pattern and form in morphogenesis, J. Math. Biol. 19:265-279 (1984).

7 L. Olsen, J. A. Sherratt, and P. K. Maini, A mechanochemical model for adult dermal wound contraction and the permanence of the contracted tissue displacement profile, J. Theor. Biol. 177:113-128 (1995).

8 R. A. F. Clark, Overview and general considerations of wound repair, In The Molecular and Cellular Biology of Wound Repair, R. A. F. Clark and P. M. Henson, Eds., Plenum, New York, 1988, pp. 3-34.

9 Y. C. Fung, Biomechanics, 2nd ed., Springer-Verlag, New York, 1993.

10 L. D. Landau and E. M. Lifshitz, Theory of Elasticity, Pergamon, New York, 1970.

11 J. D. Murray, P. K. Maini, and R. T. Tranquillo, Mechanochemical models for generating biological pattern and form in development, Physics Reports 171:59-84 (1988).

12 E. Bell, B. Ivarsson, and C. Merrill, Production of a tissuc-like structure by contraction of collagen lattices by human fibroblasts of different proliferative potential in vitro, Proc. Natl. Acad. Sci. USA, 76:1274-1278 (1979).

13 P. D. Asmussen and B. Söllner, Wound Care: Principles of Wound Healing, Beiersdorf Medical Bibliothek, Hamburg, 1993.

14 M. H. McGrath and R. H. Simon, Wound geometry and the kinetics of wound contraction, Plast. Reconstr. Surg. 72:66-72 (1983).

15 R. T. Tranquillo and J. D. Murray, Continuum model of fibroblast-driven wound contraction: inflammation-mediation, J. Theor. Biol. 158:135-172 (1992).

16 L. Olsen, J. A. Sherratt, and P. K. Maini, A mathematical model for fibroproliferative wound healing disorders, Bull. Math. Biol. 58:787-808 (1996).

17 P. Tracqui, D. E. Woodward, G. C. Cruywagen, J. Cook, and J. D. Murray, A mechanical model for fibroblast-driven wound healing, J. Biol. Syst. 3:1075-1084 (1995).

18 F. Grinnell, Fibroblasts, myofibroblasts, and wound contraction, J. Cell Biol. 124:401-404 (1994).

19 V. H. Barocas, A. G. Moon, and R. T. Tranquillo, The fibroblast-populated collagen microsphere assay of cell traction force-part 2: measurement of the cell traction parameter, J. Biomech. Eng. 117:161-170 (1995). 
20 M. Eastwood, D. A. McGrouther, and R. A. Brown, A culture force monitor for measurement of contraction forces generated in human dermal fibroblast cultures: evidence for cell-matrix mechanical signalling, Biochim. Biophys. Acta 1201:186-192 (1994).

21 H.P. Ehrlich and J. B. M. Rajaratnam, Cell locomotion forces versus cell contraction forces for collagen lattice contraction: an in vitro model of wound contraction, Tissue Cell 22:407-417 (1990).

22 B. Nusgens, C. Merill, C. Lapiere, and E. Bell, Collagen biosynthesis by cells in a tissue equivalent matrix in vitro, Collagen Relat. Res. 4:351-363 (1984).

23 J. D. Murray, Mathematical Biology, Springer-Verlag, New York, 1989. 